\title{
Phase-based features for Motor Imagery Brain-Computer Interfaces
}

\author{
Benjamin Hamner, Robert Leeb, Michele Tavella, and José del R. Millán
}

\begin{abstract}
Motor imagery (MI) brain-computer interfaces (BCIs) translate a subject's motor intention to a command signal. Most MI BCIs use power features in the mu or beta rhythms, while several results have been reported using a measure of phase synchrony, the phase-locking value (PLV). In this study, we investigated the performance of various phasebased features, including instantaneous phase difference (IPD) and PLV, for control of a MI BCI. Patterns of phase synchrony differentially appear over the motor cortices and between the primary motor cortex (M1) and supplementary motor area (SMA) during MI. Offline results, along with preliminary online sessions, indicate that IPD serves as a robust control signal for differentiating between MI classes, and that the phase relations between channels are relatively stable over several months. Offline and online trial-level classification accuracies based on IPD ranged from $84 \%$ to $99 \%$, whereas the performance for the corresponding amplitude features ranged from $70 \%$ to $100 \%$.

Index Terms - BCI, EEG, motor imagery, phase synchrony, instantaneous phase difference
\end{abstract}

\section{INTRODUCTION}

Many different electroencephalogram (EEG)-based braincomputer interfaces (BCIs) have recently been developed. In EEG-based BCIs, features are extracted from EEG signals to restore communication to patients with locked-in syndrome or to aid patients with muscular deficiencies [1]. Additionally, BCIs can be useful for healthy users in certain situations.

One type of BCI uses EEG signals that are dependent on motor imagery. A subject imagines performing a movement, such as opening and closing his hand. This results in increased power in the mu rhythm $(10-14 \mathrm{~Hz})$ over the ipsilateral motor cortex, and decreased power over the contralateral motor cortex. Most MI BCIs used spatio-spectral features, such as band power, welsh power spectrum, or common spatial patterns for classification [1], [2]. However, a few studies have showed discriminatory information exists in the signal phase as well [3], [4].

In this study we systematically examined various features based on the relative phase between mu-rhythm EEG signals offline. We propose new features to model the phase relations between EEG channels, and compare them with existing phase and amplitude-based features. Additionally, we present preliminary results from one subject online. Our goal is twofold: to improve BCI performance by incorporating addi-

This research is supported by the European ICT Programme Project FP722461 (TOBI), and B. Hamner is supported by the Whitaker Foundation. This paper only reflects the author's views and funding agencies are not liable for any use that may be made of the information contained within.

B. Hamner, R. Leeb, M. Tavella and J.d.R. Millán are with the Defitech Chair on Non-Invasive Brain-Machine Interface, Center for Neuroprosthetics, School of Engineering, Ecole Polytechnique Fédéral de Lausanne, Lausanne, Switzerland. benjamin.hamner@epfl.ch jose.millandepfl.ch tional information offered by signal phase, and to enhance our understanding of the synchronization between brain regions during mental tasks.

\section{METHODS}

\section{A. Experimental Setup}

Previously trained subjects delivered mental commands through an EEG-based BCI. To control our two-class BCI, the subjects learned to voluntarily modulate EEG rhythms by performing two of the following three MI tasks: imagined movement of the left hand $(\mathrm{LH})$, right hand $(\mathrm{RH})$, or both feet $(\mathrm{BF})$. In the experimental protocol, a fixation cross was displayed at the start of a trial. A second later, the left or right cue was displayed. Subjects were instructed to begin the corresponding MI task at this point. One second later, continuous feedback started and the subject attempted to move the feedback bar to the corresponding side of the screen. Once the bar passed a threshold on either side, the command was displayed for a second, and then the bar was reset. A second later the next trial started.

A total of four male healthy subjects (A6, B3, C9, and D7; 23-28 years old) were studied for this experiment. A6 used BF-RH MI to control the BCI, while the rest used LHRH MI. For each subject, three sessions were recorded for training, and then three sessions were recorded for testing. Testing sessions were recorded between 56 and 75 days after training sessions. For both sets of data, subjects used a statistical Gaussian classifier on power spectral density features, as described in [5]. EEG was recorded with a 16channel gUSBamp active electrode system at $512 \mathrm{~Hz}$. The hardware filter was set to $0.1-100 \mathrm{~Hz}$ and the notch filter was on. The following EEG channels were recorded: $\mathrm{Fz}$, FC3, FC1, FCz, FC2, FC4, C3, C1, Cz, C2, C4, CP3, CP1, $\mathrm{CPz}, \mathrm{CP} 2$, and $\mathrm{CP} 4$.

No trials were discarded. This resulted in 45 trials of each MI class for the training period and 45 trials of each MI class for the testing period, for a total of 180 trials per subject.

\section{B. EEG Preprocessing}

The EEG data was preprocessed using a discrete laplacian spatial filter and then filtered with a $n^{t h}$-order causal Butterworth bandpass filter, $n \in\{2,4,6,8\}$. The optimal cutoff frequencies were selected for each subject based on the training data.

\section{Analytic Signal Method}

There are two methods to extract the phase from an arbitrary signal, the analytic method and a wavelet-based method. According to [6], these are approximately the same 
for EEG signals, and we used the analytical signal method. Given a signal $x(t)$, the analytic signal $x_{a}(t)$ is defined as

$$
x_{a}(t)=x(t)+i \tilde{x}(t)=A_{x}(t) e^{i \theta_{x}(t)},
$$

where $\tilde{x}(t)$ is the Hilbert transform of $x(t)$. The instantaneous phase $\theta_{x}(t)$ is calculated as follows:

$$
\theta_{x}(t)=\operatorname{Arg}\left(x_{a}(t)\right)
$$

\section{Features}

Two types of features were extracted from the analytic EEG signals: instantaneous features were calculated independently at each time point, and all other features were calculated over a $T$-sample window prior to the current instant. For this study, $T=512$ samples (1 second). Successive windows were one sample apart.

1) Instantaneous Phase Difference (IPD): The instantaneous phase difference $\phi_{x y} \in[-\pi, \pi)$ was calculated between each pair of EEG channel $(x, y)$ instantaneous phases, $\theta_{x}$ and $\theta_{y}$. It was calculated as,

$$
\phi_{x y}=\left[\begin{array}{ll}
\theta_{x}-\theta_{y}+\pi & (\bmod 2 \pi)
\end{array}\right]-\pi
$$

2) Phase Locking Value (PLV): The phase locking value is a measure of the synchronization between pairs of signal. It is calculated over the $T$-sample window as follows:

$$
P L V=\left|\frac{1}{T} \sum_{t=1}^{T} e^{j \phi_{x y}}\right|
$$

PLV $\in[0,1]$, where 0 represents completely desynchronized signals and 1 represents completely synchronized signals [3].

3) Mean Phase Difference (MPD): The mean phase difference was defined to be the mean of the IPD between a pair of narrowband EEG signals over the time window. It was computed as follows:

$$
M P D=\operatorname{Arg}\left(\sum_{t=1}^{T} e^{j \phi_{x y}}\right)
$$

4) Instantaneous Amplitude (IA): The instantaneous amplitude was calculated as the magnitude of the analytic signal:

$$
I A=\left|x_{a}(t)\right|
$$

5) Average Posterior (AP) of Instantaneous Features: To compare the instantaneous features (IA and IPD) with the windowed features, the mean of the posterior probabilities output from probabilistic classifiers based on IA and IPD was computed over the window length as well. This generated two new corresponding features, IA-AP and IPD-AP.

\section{E. Univariate Probability Distributions}

A parametric univariate probability distribution was fit to each feature. IPD and MPD were modeled with wrapped Cauchy distributions, IA was modeled with a Rician distribution, and PLV was modeled with a beta distribution. Maximum Likelihood Estimation (MLE) was used to estimate the parameters of each distribution [7], [8].

\section{F. Feature Selection}

For the IA features, all 16 channels were used. For the IPD, MPD, and PLV features, univariate marginal distributions were fit for each of the 120 possible channel pairs. The 16 channel pairs with the highest expected values of the posterior correct class on the training set were used.

\section{G. Classification}

A Naive Bayes (NB) classifier was analyzed for its flexibility in selecting the parametric model for the marginal distributions. This classifier is limited by its feature independence assumption, but we were initially interested in generatively modeling and comparing the features themselves as opposed to various supervised machine learning techniques.

\section{H. Evaluation}

Posterior class probabilities were calculated for every sample (at $512 \mathrm{~Hz}$ ) on the test set, and feature sets and classifiers were compared according to class-balanced accuracy (BACC). Samples from the start of continuous feedback until the feedback bar crosses the threshold were considered for evaluation. To compute the BACC, the percentage of correctly identified samples within each class was determined, and then these were averaged across the classes. Since there are two classes, a BACC of $50 \%$ is random performance, and $100 \%$ is perfect performance. We focus on the sample-level performance since these posterior probabilities control the direction of bar movement in the immediate feedback given to the user.

We also evaluate the trial-level classification performance, where posterior probabilities are averaged over the trial, and the class with the maximum average posterior is determined.

\section{RESULTS}

Fig. 1 shows the differential amplitudes (IA) and phase synchronies (PLV) that occur between motor imagery classes. For each of the LH-RH subjects, the amplitude of the mu rhythm shows a relative increase over the ipsilateral motor cortex and decrease over the contralateral motor cortex, as expected. These subjects have an increase in the phase synchrony over the ipsilateral motor cortex and between the ipsilateral motor cortex and the frontal areas, and a decrease for the corresponding contralateral areas as well. The BF-RH subject showed a relative decrease in power over the contralateral motor cortex and increase in power over the central areas. BF MI resulted in a relative increase in synchrony over the left motor cortex and a broad relative decrease in phase synchrony elsewhere, in comparison with RH MI.

All subjects showed changes between the PLV of the Fz channel and channels over M1, though only subject A6 showed significant amplitude changes in the Fz channel. This indicates synchronization between the SMA and M1 during MI tasks. Also, all subjects tended to display synchronization within medial and ipsilateral locations or between medial and lateral channels, but there were little changes in phase 

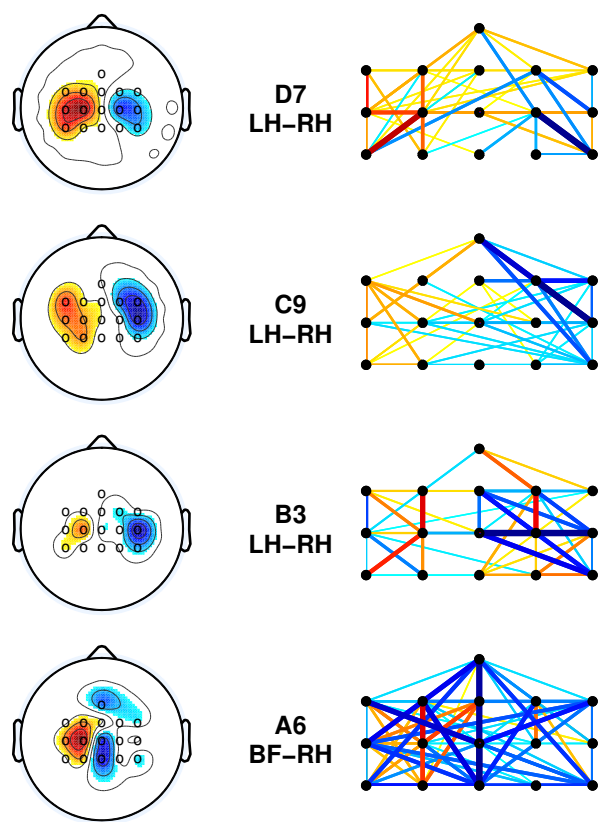

Fig. 1. The figure shows the differential amplitudes and phase synchronies that occur in the mu rhythm band between different motor imagery classes for each subject. The left column displays the mean relative changes in IA and the right column displays the mean changes in PLV across the entire training period. Red indicates an increase in power or phase synchronizations from $\mathrm{LH} / \mathrm{BF}$ MI relative to $\mathrm{RH}$ MI, whereas blue indicates a relative decrease in power or phase synchronizations. In the right column, the thickness of the line corresponds to the magnitude of the differential PLV.
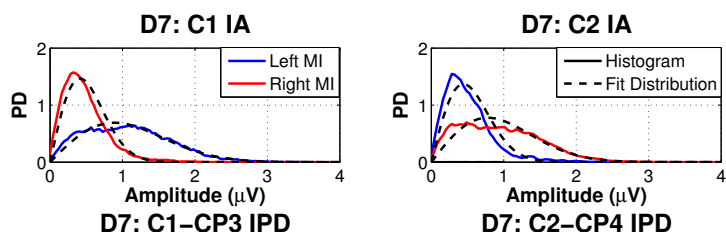

D7: C2-CP4 IPD
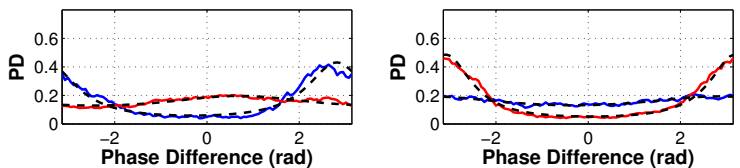

D7: C1-CP3 MPD

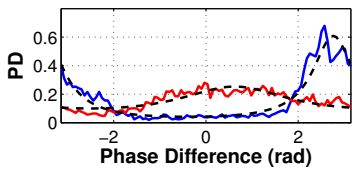

D7: C2-CP4 MPD

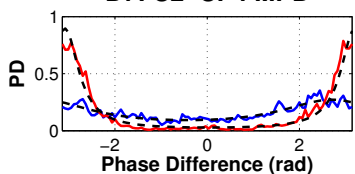

D7: C1-CP3 PLV

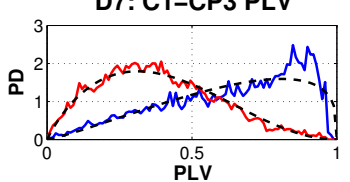

D7: C2-CP4 PLV

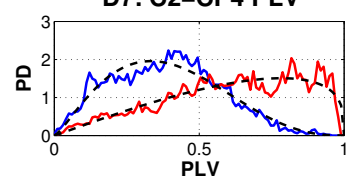

Fig. 2. The figure shows examples of the probability densities (PD) of histograms and parametric distributions for the IPD, MPD, PLV, and IA features from D7's training data. The IA is shown for the two most discriminant electrode channels, $\mathrm{C} 1$ and $\mathrm{C} 2$, and the phase features are shown for the two most discriminant channel pairs, C1-CP3 and C2-CP4 A $2^{\text {nd }}$-order Butterworth BPF was used.
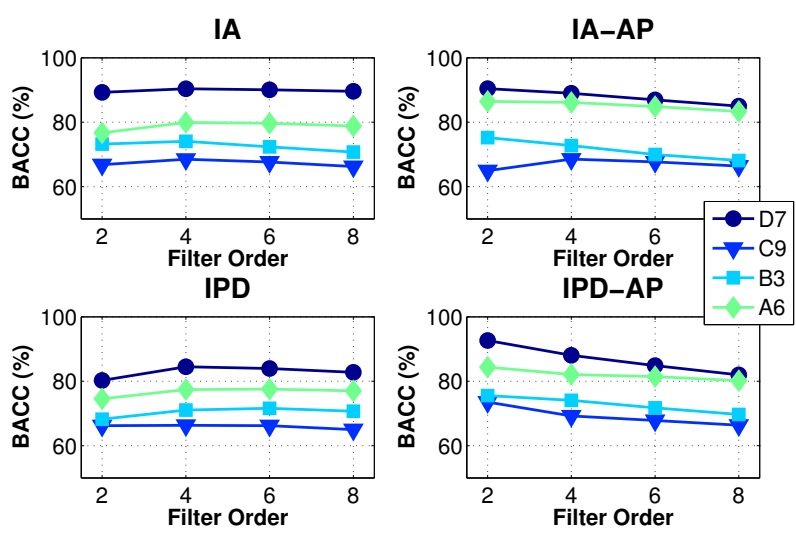

IPD-AP

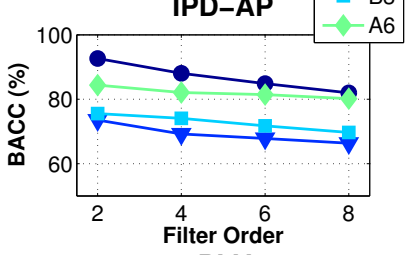

MPD
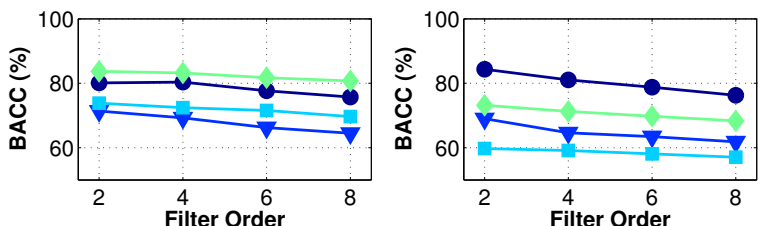

Fig. 3. The figure shows the effect of filter order on NB classification performance at the sample level. The six plots represent the six features, and the color and marker signify the subject.

synchronization observed between channels over contralateral areas of the brain. Fig. 2 shows examples of the fit univariate marginals for examples of the IA, IPD, MPD, and PLV features. All of the fit distributions closely approximate the histograms, with one slight exception. The histogram for the C1-CP3 PLV feature is slightly too skewed for a beta distribution under LH MI. In general, the PLV features only had beta distributions if the filter order was sufficiently low or the time windows were sufficiently long. Also, both IPD and MPD features displayed show synchrony during one class of MI and are desynchronized during the other class. Other IPD and MPD features show similar levels of synchrony for both classes, but are synchronized to opposite relative phases.

Fig. 3 shows the effect of filter order on classification performance for the IPD and IA features, along with IPDAP and IA-AP. For the instantaneous features, a $4^{t h}$-order filter results in the highest classification accuracy for each subject. For the windowed versions of these features, a $2^{\text {nd }}$ order filter results in the highest classification performance, with one exception. This yields two clear trends: performance with instantaneous features peaks at a $4^{\text {th }}$ order filter, and performance with windowed features deteriorates with increasing filter order. These trends had two causes. First, increasing the filter order increases the delay introduced in the signal, from about $0.1 \mathrm{~s}$ for a $2^{\text {nd }}$-order filter to $0.5 \mathrm{~s}$ for an $8^{t h}$ order filter. This made samples close to the onset of motor imagery harder to classify at higher filter orders. For the instantaneous features, estimates of signal amplitude and phase were noisier with the lowest-order filter, which resulted in the $4^{\text {th }}$-order filter having peak performance. Secondly, increasing the filter order effectively smoothed the extracted 

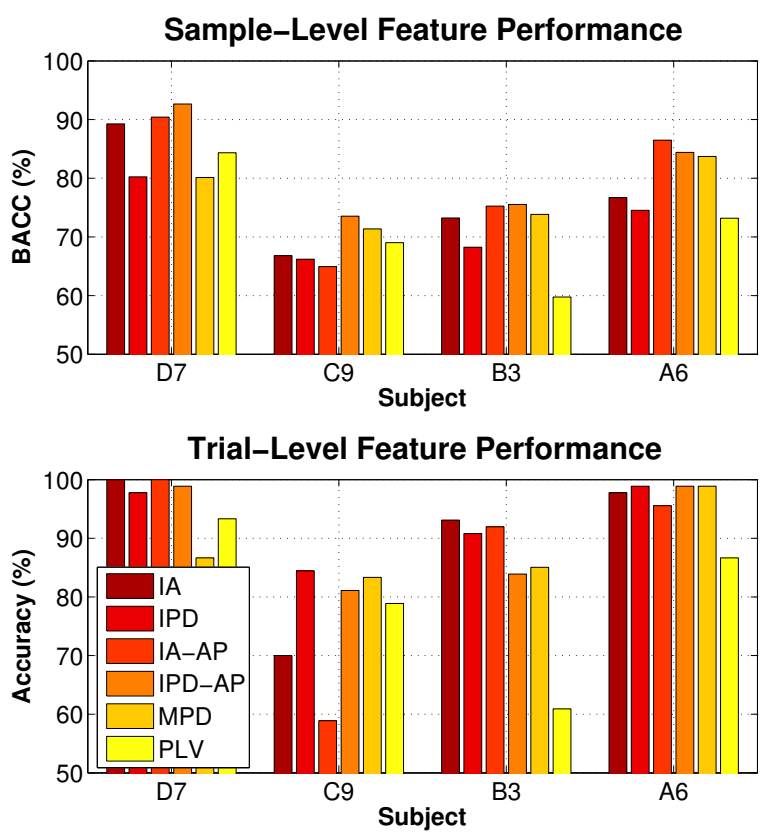

Fig. 4. The top plot shows sample-level classification performance in BACC with $2^{\text {nd }}$-order BPFs. The bottom plot shows trial-level classification performance with $2^{\text {nd }}$-order BPFs.

features across time. This meant that the amplitudes and phase differences within a window were more correlated for higher filter orders, reducing the benefit of using a timewindow and lowering classification performance.

Fig. 4 shows the sample-level and trial-level performance of different features with NB on the testing sessions. There are a number of clear trends across subjects. On the sample level, the windowed versions of the instantaneous features (IA-AP) and (IPD-AP) perform better than the corresponding instantaneous features (IA and IPD). IPD-AP also performs much better than the PLV feature. The relative performance of MPD and PLV is ambiguous: for two subjects they are similar, and for two MPD is much better. For three subjects, IPD-AP performed similarly (within 2\%) to IA-AP. Subject C9 saw a 9\% improvement in BACC between IA-AP and IPD-AP. On the trial level, classification performance for IPD ranged from $84 \%$ to $99 \%$ correct, whereas classification performance for IA ranged from $70 \%$ to $100 \%$. One subject saw a dramatic improvement with the IPD feature, with 10 more trials classified correctly than IA. Trial-level performance of the windowed features tends to be slightly lower than the corresponding instantaneous features, as the windowed features incorporate some information prior to the start of the continuous feedback.

Additionally, three preliminary online sessions were run with subject D7. A NB classifier was used on two features, the C1-CP3 and C2-CP4 IPDs. D7 had good control of the feedback bar with the IPD features, and completed 89 out of 90 trials correctly.

\section{CONCLUSIONS}

In this paper we have demonstrated that the instantaneous phase differences (IPD) between narrowband EEG signals performs competitively with power features in controlling a MI BCI. This performance was confirmed online with three preliminary sessions on one subject. During these sessions, a 99\% classification performance was achieved. Additionally, the IPD-AP feature perform significantly better than the PLV features reported in literature. This was because the IPDAP feature incorporated information on both the instantaneous phase difference of the signals and their synchrony across time, whereas the PLV feature only measured their synchrony.

As a next step, we will conduct a study that compares the online performance of power-based features, phase-based features, and the combination of the two. This has the potential to enhance the performance of subjects that already have good BCI control and to help other subjects gain control.

Though the NB classifier allowed us to flexibly choose the univariate marginal distributions, it was limited by its feature independence assumption. There are correlations within and between both the amplitude-based and phase-based features. In the future we will investigate methods to directly model these dependencies.

Accurately modeling the amplitude and phases we observe in EEG signals has a threefold advantage: it will potentially aid in a faster determination of mental state, allow us to differentiate between a greater number of states, and improve our understanding of the underlying neurophysiological processes.

\section{REFERENCES}

[1] J.R. Wolpaw, N. Birbaumer, D.J. McFarland, G. Pfurtscheller, and T. M. Vaughan, "Brain-computer interfaces for communication and control" Clin. Neurophysiol., vol. 113, pp. 767-791, 2002.

[2] H. Ramoser, J. Muller-Gerking, and G. Pfurstchller, "Optimal spatial filtering of single-trial EEG during imagined hand movement", IEEE Trans. Rehabil. Eng., no. 8, pp. 441-446, 2000.

[3] Y. Wang, B. Hong, X. Gao, and S. Gao, "Phase Synchrony Measurement in Motor Cortex for Classifying Single-trial EEG during Motor Imagery", Proc. of the 28th IEEE EMBS Annual Int. Conf., New York City, NY, pp. 75-78, 2006.

[4] C. Brunner, R. Scherer, B. Graimann, G. Supp, and G. Pfurtscheller, "Online Control of a Brain-Computer Interface Using Phase Synchronization", IEEE Trans. on Biomed. Eng., vol. 53, no. 1, 2006.

[5] J. d. R. Millán, P. Ferrez, F. Galán, E. Lew, and R. Chavarriaga, "Noninvasive brain-machine interaction," Int. J. Pattern Recognit. Artif. Intell., vol. 22, pp. 959-972, 2008.

[6] M. Le Van Quyen, J. Foucher, J.P. Lauchaux, E. Rodriguez, A. Lutz, J. Martinerie, and F. Varela, "Comparison of Hilbert transform and wavelet methods for the analysis of neuronal synhrony", J. Neurosci. Methods, vol. 111, pp. 83-98, 2001.

[7] J.T. Kent and D.E. Tyler, "Maximum likelihood estimation for the wrapped Cauchy distribution", J. of Applied Statistics, vol. 14, iss. 2, pp. 247-254, 1988.

[8] J. Sibjvers, A.J. den Dekker, P. Scheunders, and D. Van Dyck, "Maximum Likelihood estimation of Rician distribution parameters", IEEE Trans. on Medical Imaging, vol. 17, nr. 3, pp. 357-361, 1998. 